\title{
The Amazing Variety of Public Math
}

\section{David Eisenbud and Kirsten Bohl}

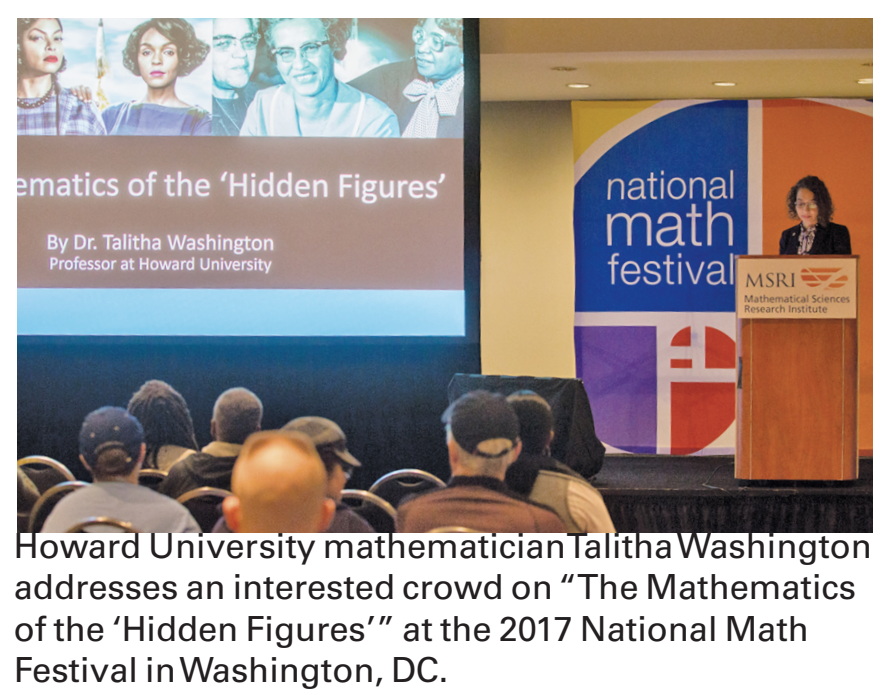

Many of us who believe passionately that math belongs to everyone used to think of math outreach as puzzles and games; media coverage such as books, articles, or films highlighting some math or a math personality in an accessible way; or art and design, showcasing beauty in two or three dimensions.

Those things are wonderful. But as we at MSRI have developed the programming for the National Math Festival (NMF) - appearing in its third incarnation on Saturday, May 4, 2019 at the Washington DC convention center-we have been amazed at the breadth of the ways math can

David Eisenbud is Director of the Mathematical Sciences Research Institute (MSRI) and Professor of Mathematics at the University of California, Berkeley. His email address is de@msri . org.

Kirsten Bohl is the coordinator of the National Math Festival. Her email address is kboh1@msri.org.

For permission to reprint this article, please contact: reprint -permission@ams.org.

DOI: https://dx.doi.org/10.1090/noti 1875 be appreciated by the public. Hoping that others will see how to realize even more variety, we list some of what will happen at the NMF this year (all different from the events in past versions):

Among twenty-one distinct stage presentations and performances will be:

- A world-class New York dance ensemble with Dance of the Diagram, commissioned by Jim Simons to highlight the beauty of differential cohomology;

- A recent winner of the "Dance Your PhD" contest (AAAS and Science magazine), Nancy Scherich, to share her insights on braiding and anchoring a math Maypole;

- A whirlwind tour of 300 years of mathematical and musical history with Lillian Pierce-complete with a violin solo;

- Marcus du Sautoy's reflections on creativity and artificial intelligence;

- Francis Su's exploration of The Mathematics of Human Flourishing;

- Holly Krieger's peek into prime numbers, by way of the YouTube channel Numberphile (2.7 million subscribers and counting...);

- Former Baltimore Ravens player John Urschel's sharing of his enthusiasms for the math of chess and the math of football; and

- An opportunity to touch a prehistoric ice core, accompanied by a talk for ages $8+$ about 


\section{COMMUNICATION}

the mathematics of sustainability, with Mary Lou Zeeman and the Mathematics of Planet Earth team.

The National Science Foundation will screen the winners of its first-ever "We Are Mathematics" short film contest; Joseph Teran will draw a young audience with clips from his team's animation work in Frozen and Moana; and MSRI will screen a trailer of the forthcoming public television film about the inspiring, joyous life of Maryam Mirzakhani. All of this takes place in the Alfred P. Sloan Foundation Film Room.

Many organizations besides MSRI work hard and successfully to encourage math appreciation, and the NMF will give them a platform, too. New to the Festival, the Make or Take Spiral is not your regular exhibit setup $\left(10^{\prime} \times 10^{\prime}\right.$ booths with skirted $\sigma^{\prime}$ tables). Instead, it is a large Fibonacci-spiral-shaped structure with interior rooms housing about 20 different math organizations who individually and collectively will mobilize Festival-goers to "take math home with them" in a wide variety of ways. For example:

- The National Council of Teachers of Mathematics (NCTM) will bring tips and resources for classroom teachers and homeschool parents.

- The Erikson Institute Early Math Collaborative and DREME Network out of Stanford University will bring ideas, energy, and resources for preschool families.

- The National Science Foundation will bring two scavenger hunts, one for children and one for adults, both designed to showcase just how much math research they support.

- Four local Math Circles will come ready to sign up families for the next academic year.

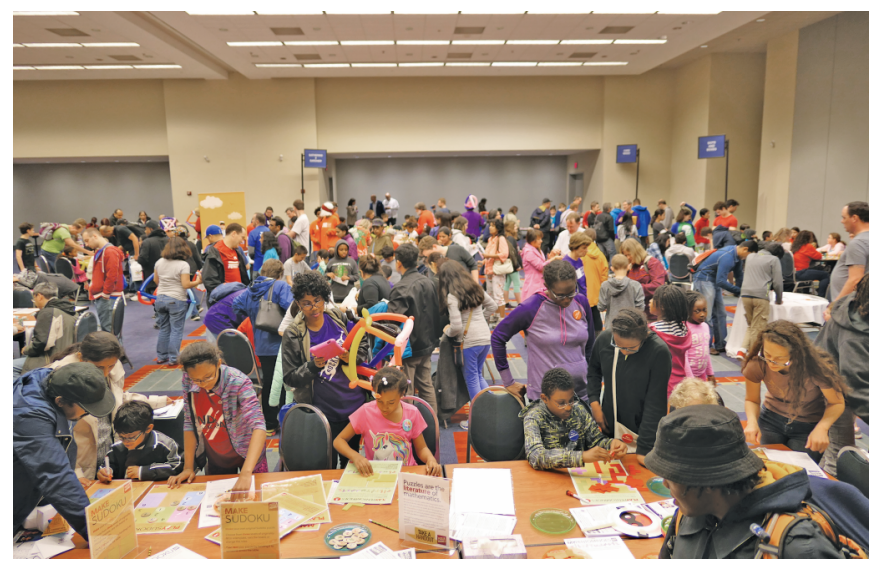

Families enjoy tabletop activities hosted by the Julia Robinson Mathematics Festival in what was colloquially known as "The Games and Puzzles Room," NMF 2017 Celebration of Mind.

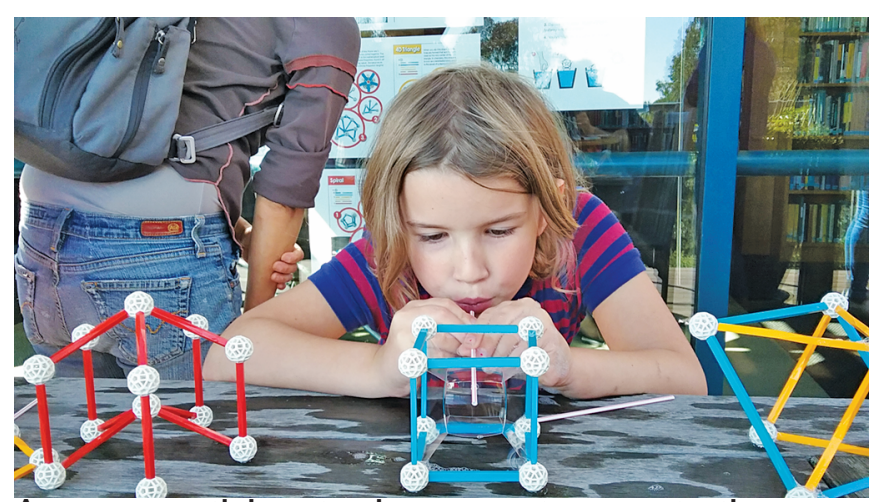

A young participant evinces great concentration while inserting another bubble inside a Zometool frame at a Geometric Bubble Blowing event. Museums around the country will host such events in May 2019.

Along with the novelties mentioned above, games and puzzles are a staple of public interest in math, and they will be well represented. New to the 2019 Festival is a newly released collection of board games for family-style play called South of the Sahara, created by the MIND Research Institute. Drawing on ancient math games from Ghana, Mozambique, and Madagascar, these games are available in English and Spanish, best for ages 7 and up. Many more games will make an appearance at the Festival...including a chance to bring along and share your own favorite maththemed board games.

The National Math Festival has also catalyzed associated events around the country. Bubbles and soap films are always a source of wonder, and dozens of science museums around the US will host events on the same day as the Festival. With the support of the construction-set maker Zometool $^{\circledR}$, member museums affiliated with the Association of Science-Technology Centers (ASTC) will offer hands-on bubble blowing fun for all ages. Participants will dip Zometool wands of three-dimensional shapes such as cubes or tetrahedra into buckets of a homemade "bubble goop" made with Dawn ${ }^{\circledR}$ dish soap, and explore what happens when the wand is later shaken, or an additional bubble is deposited into the center of the frame with a straw, and so on. For starters, the geometric shape of the bubble inside the structure is often quite beautiful, and also not what one might expect. These events will serve as an amplification of the NMF on Saturday, May 4.

The world of insightful online recreational math content for families continues to amaze as well, and we salute its expansion with an ever-growing but carefully chosen set of books, videos, puzzles, games, and toys sortable by age appropriateness (ages 2-18+), in the More Math! section of the NMF web site Nationa 1MathFestiva1 . org.

The 2019 National Math Festival is organized by the Mathematical Sciences Research Institute (MSRI) in cooperation with the Institute for Advanced Study (IAS) and 


\section{COMMUNICATION}

the National Museum of Mathematics (MoMath). We are grateful to the many dedicated mathematicians and math organizations-such as the AMS, AWM, Bridges, Julia Robinson Mathematics Festival, NAM, NOVA, The Young People's Project, and many, many others-who will bring this large-scale event to life.

We can only look forward to yet more surprises in this wonderful landscape of public engagement!

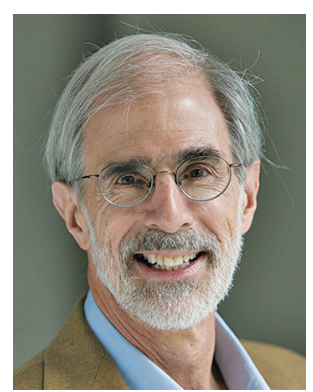

David Eisenbud

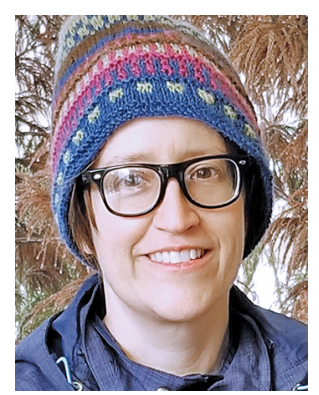

Kirsten Bohl

\section{Credits}

Photo of David Eisenbud is courtesy of the Simons Foundation.

Photo of Kirsten Bohl is by Randell Christopher.

Photo of Talitha Washington is by Lexey Swall.

Photo of 2017 NMF Celebration of Mind is by David Eisenbud.

Photo of Geometric Bubble Blowing is by Kirsten Bohl.

\section{Advertise in the}

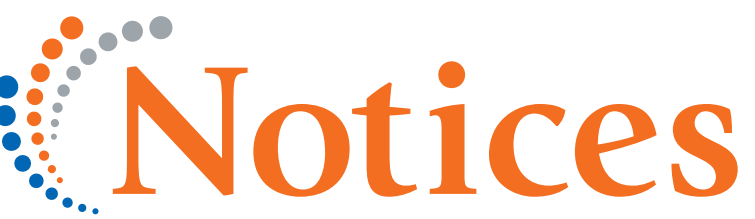

of the American Mathematical Society

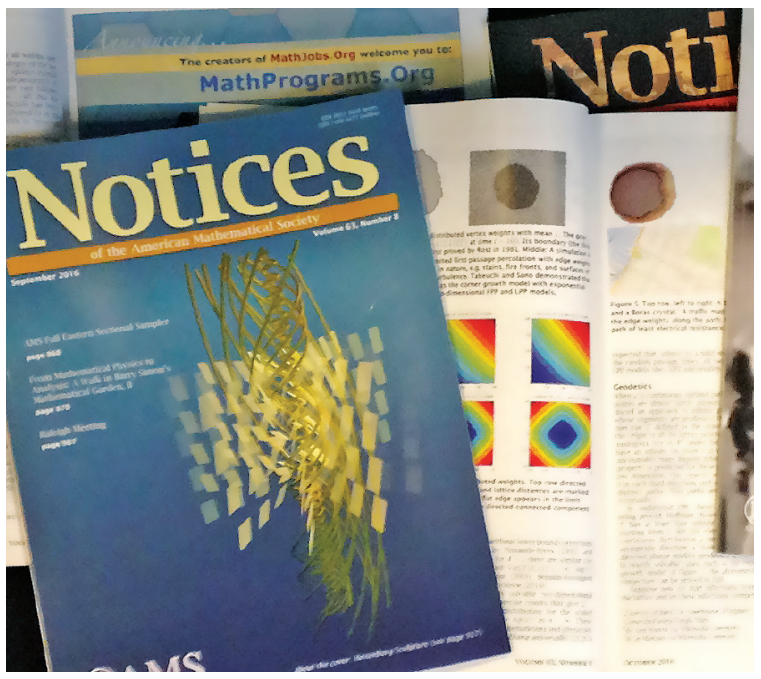

Connect with an audience of approximately 30,000 subscribers through Classified and Display Advertising in the Notices of the American Mathematical Society, available in print and online.

As the world's most widely read magazine aimed at professional mathematicians, the Notices is an excellent medium for announcing publications, products, and services, and for recruiting mathematical scientists in academic, industry, and government.

\section{Learn more about advertising} in the Notices at www.ams.org/noticesadvertising.

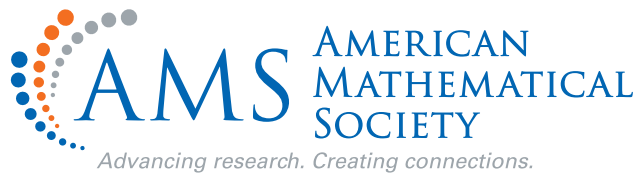

